International Journal of Engineering \& Technology, $7(3.13)(2018) 61-67$
International Journal of Engineering \& Technology
SPC
Website: www.sciencepubco.com/index.php/IJET
Research paper

\title{
Robust Diagnosis of Vehicular Active Suspension System Using Bond Graph Approach
}

\author{
Abderrahmene Sellami ${ }^{1 *}$, Dhia Mzoughi ${ }^{2}$, Abdelkader Mami ${ }^{3}$ \\ ${ }^{1-3}$ Analysis, Design and Control Systems Laboratory-ENIT, \\ Faculty of Sciences of Tunis, Tunis El Manar University, Tunis, Tunisia \\ *Corresponding author E-mail: abderrahmenesallami@gmail.com
}

\begin{abstract}
Diagnostic systems play a major role in the safety of industrial systems and the availability of its equipment. Rapid detection (as soon as possible) to the operator of the detected deviations (defects) in relation to the expected nominal behavior is fundamental for the implementation of preventive and corrective actions on industrial systems.

These industrial systems are governed by several physical phenomena and various technological components, which is why the Bond Graph tool, based on an energy and multi-physical analysis, is well suited. In this article, we will discuss the problem of diagnosing an active suspension system of a vehicle by presenting diagnostic methods. Then, we introduce the bond graph tool for the robust diagnosis of the system. Finally, the extension of the deterministic models presented to models integrating uncertain elements (Fractional linear transformations LFT) and the generation of robust analytical redundancy relationships are also detailed.
\end{abstract}

Keywords: Robust Diagnosis, Bond Graph, Vehicular Active Suspension System, Fractional Linear Transformations.

\section{Introduction}

In an industrial system, the slightest failure is catastrophic in an environment where performance is paramount. For this, it is evident to ensure that the process is functioning properly in relation to the objectives that have been summoned to it [1-6].

The information to translate the behavior of a system is given by the measurements of the variables of it. Indeed, the quality of the measurements is an essential element to allow the system of diagnosis and the evaluation of the performances of a process. The quality of the information can be increased by improving the accuracy of the instrumentation and by multiplying the number of sensors. For technical or financial reasons, this solution is reserved for high-tech industries or those with high technological risks. Moreover, this hardware redundancy does not make it possible to protect against a failure of certain common elements of the measurement chain [7-12].

Moreover, analytical redundancy has the advantage of not increasing the cost of the installation and freeing itself from the hardware constraints. In the field of diagnostics, methods based on the concept of information redundancy have been developed. Their principle is generally based on a test of coherence between an observed behavior of the process provided by sensors and an expected behavior provided by a mathematical representation of the process.

Analytical redundancy methods therefore require a model of the system to be monitored. This model includes a number of parameters whose values are assumed to be known during normal operation. The comparison between the actual behavior of the system and the expected behavior given by the model provides a quantity called the residue that will be used to determine whether the system is in a failing state and to specify the part or component of the system faulty system [13-18].
Automation engineers consider the Bond Graph approach as the most reliable approach among other approaches to monitoring industrial plants. This reliability is given not only the modeling and the simulation but also the certain and uncertain diagnosis. In this article, we are interested in the methods of certain and uncertain diagnosis based on the bond graph model. At the beginning, the definite approach is presented by the generation of the analytic redundancy relations using the leap graph approach. In the following, the extension of the deterministic models presented to models integrating uncertain elements (Linear Fractional Transformations LFT) and the generation of robust analytical redundancy relationships (ARRs) are also detailed in this paper. Finally, a general conclusion and perspectives are given [19-22].

This article is organized as follows:

- Section 2: The first part presents a reminder on the modelling of industrial systems by the bond graph approach; the second part is devoted to the robust diagnosis of industrial systems by the leap graph approach.

- Section 3: This section is devoted to the implementation of the proposed method on an active vehicle suspension system.

- Section 4: This section presents a conclusion of this work.

\section{Robust Diagnosis by Bond Graph}

\subsection{Bond Graph Model}

The Bond Graph model of a physical system explicates the power exchanges (symbolized by links) intervening between different elements (represented by nodes) that produce, dissipate, store or transmit energy. At each node of the Bond Graph is associated one or two characteristic relations of the physical phenomenon symbolized. We thus obtain directly a set of relations between the 
magnitudes force and flux used to parameterize the physical system.

The notion of power is described by the following relation:

$$
P(t)=\dot{e}(t) \cdot f(t)
$$

This equation illustrates energy transfers in the system by using power bonds. A power link [23] is symbolized by a half arrow, whose orientation indicates the direction of power transfer. Thus, figure 1 shows the power transfer from subsystem $\boldsymbol{S}_{\boldsymbol{I}}$ to subsystem $S_{2}$.

Each power link carries two information's simultaneously: the effort and the flow (see figure 1). These are the generalized power variables (their product being the transferred power).

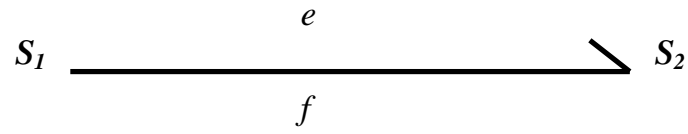

Fig.1: Power link

\subsection{Fractional Linear Transformations Model}

Fractional linear transformations (LFT) are generic objects widely used in the modeling of uncertain systems. The universality of fractional linear transformations is due to the fact that any rational expression can be written in this form [24-30]. This form of representation is widely used for the synthesis of control laws of uncertain systems using the principle of $\mu$-analysis. It consists of separating the nominal part of a model from its uncertain part as shown in figure 2. The nominal values are grouped together in an augmented matrix denoted $\mathrm{P}$, supposed to be proper, and the uncertainties whatever their type (structured and unstructured parametric uncertainties, modeling uncertainties, measurement noises, etc.) are combined in a matrix $\Delta$ of structure diagonal shown in figure 2 .

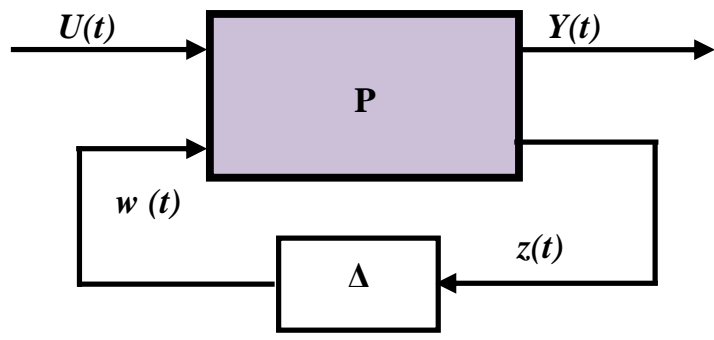

Fig. 2: Representation LFT

\subsection{Construction of a Model BG-LFT}

All industrial systems can be modeled by a hop graph model according to figure 3 . Indeed, the input signal is modeled by a source of effort $(\mathrm{Se})$ or a source of the flux $(\mathrm{Sf})$, the whole system is Modeled by resistive elements $(R)$ and storage elements $(I$ or $C)$ while the detectors are modeled by elements of the detectors $(D e$ or $D f$ ).

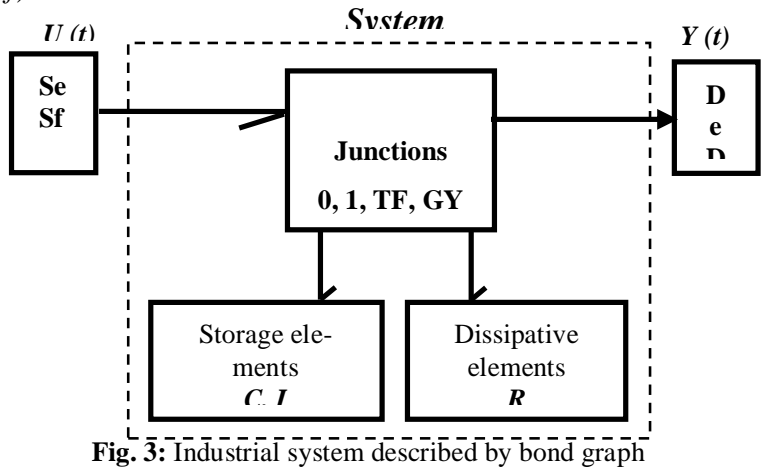

Full BG-LFT can then be represented by the diagram in figure 4 .

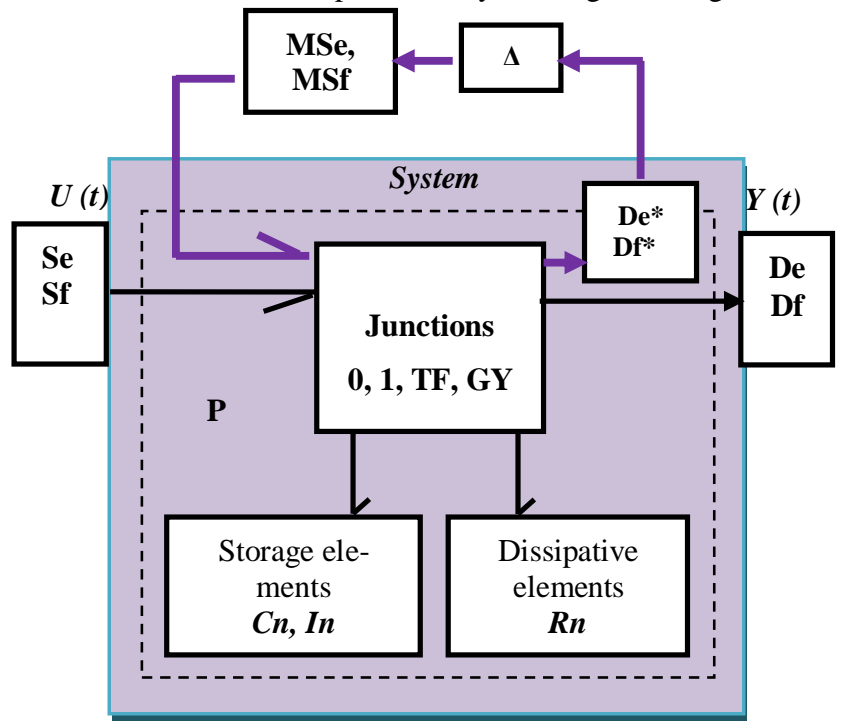

Fig. 4: Industrial system described by bond graph using LFT

\subsection{Generate Robust Residuals}

The generation of robust analytical redundancy relations from a clean bond graph model, observable and over determined is summarized by the following steps:

- $1^{\text {st }}$ step: Checking the status of the coupling on bond graph deterministic model derived preferential causality; if the system is over determined, then continue the following steps;

- $2^{\text {nd }}$ step: The bond graph model is made into LFT;

- $3^{\text {rd }}$ step: The symbolic expression of analytical redundancy relationships (ARRs) is inferred from equations junctions. This first form will be expressed by:

$\checkmark \quad$ For a junction 0:

$\sum b_{i} \mathrm{f}_{\mathrm{inc}}+\sum \mathrm{Sf}+\sum \mathrm{w}_{\mathrm{i}}$

$\checkmark \quad$ For a junction 1:

$\sum b_{i} e_{i n c}+\sum S e+\sum w_{i}$

With the sum of sources flows due to the junction 0 , the sum of the flow sources related to junction $1, b= \pm 1$ depending on whether the half-arrow into or out of the junction and $e_{i n c}$ and purpose are unknown variables.

- $4^{\text {th }}$ step: The unknown variables are eliminated by browsing the causal paths between sources and detectors or unknown variables;

- $5^{\text {th }}$ step: After removing the unknown variables, are uncertain as ARRs (5):

$R R A: \Phi\left(\sum \mathrm{Se}, \sum \mathrm{Sf}, D e, D f, \widetilde{D} e, \widetilde{D} f\right.$,

$\left.\sum w_{i}, R_{n}, I_{n}, C_{n}, T F_{n}, G Y_{n}\right)$

Or:

- $T F_{n}$ and $G y_{n}$ are nominal elements $T F$ and $G Y$;

- $R_{n}, C_{n}$ and $I_{n}$ are nominal elements $R, C$ and $I$;

- $\sum w_{i}$ is the sum of modulated inputs corresponding to uncertainties on the junction-related items. 


\section{Vehicular Active Suspension}

Consider the vehicular active suspension system and its bond graph model given in figure 4 .
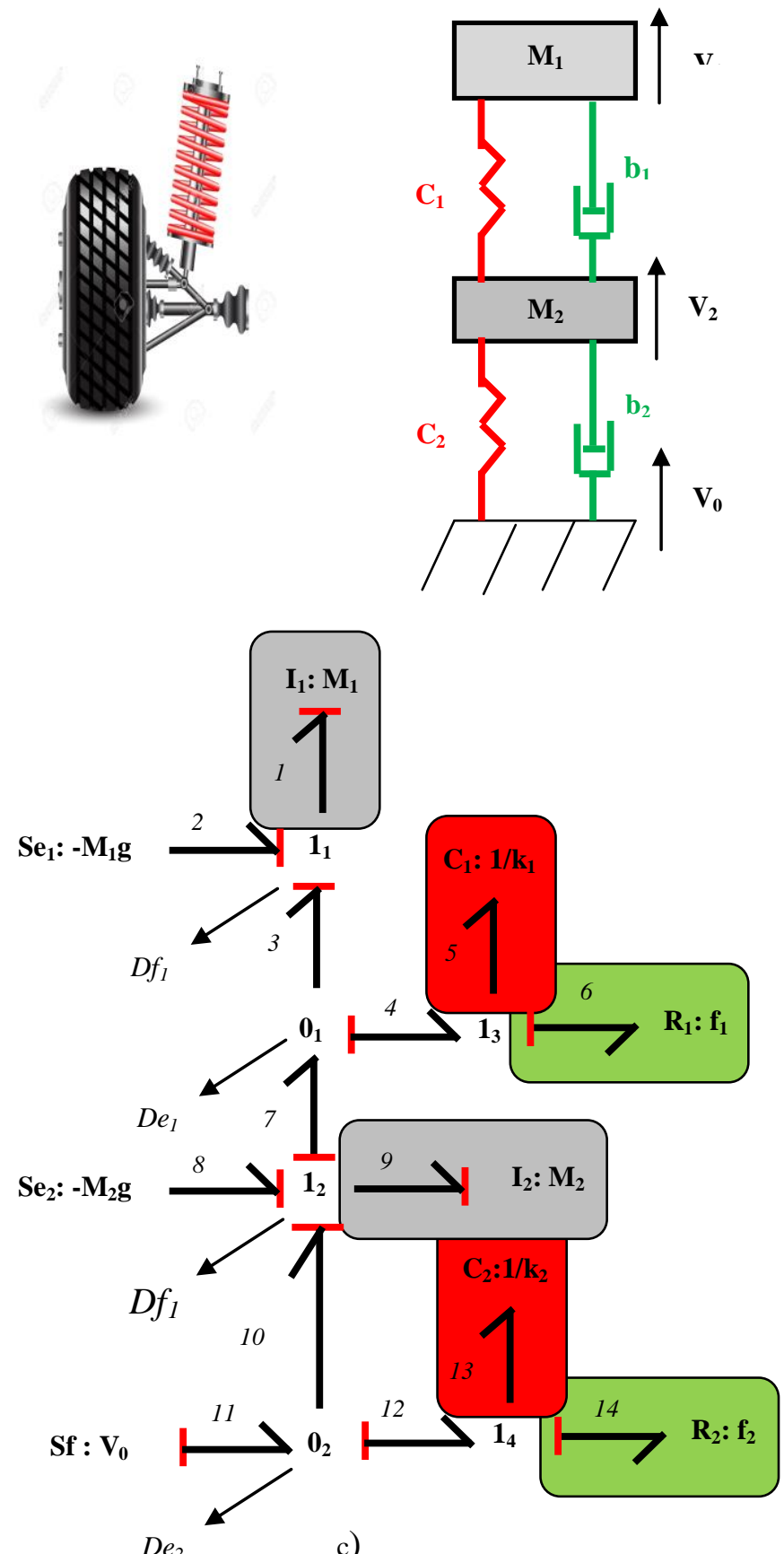

Fig. 5 : a) Real system vehicular active suspension, b) Mechanic model, c) Bond graph model

The vehicular active suspension system shown in the figure $5 \mathrm{~b}$ ) is modeled by bond graph in figure $5 \mathrm{c}$ ) as follows:

- The mass of the vehicle frame is modeled by inertial element (I: $\left.M_{1}\right)$;

- The mass of the wheel is modeled by inertial element $\left(I: M_{2}\right)$

- The Shock of the vehicle is modeled by resistive element $\left(R: f_{l}\right)$;

- The damping of the tire is modeled by resistive element $\left(R: f_{2}\right)$;

- The spring rate of the vehicle is modeled by potential element $\left(C: 1 / k_{1}\right)$;

- The spring rate of the tire is modeled by potential element $(C$ : $\left.1 / k_{2}\right)$

\subsection{Simulations of the Vehicular Active Suspension Sys-} tem

Figure 6 shows the evolution of the speed on the force exerted on the mass $(M)$ and the forces exerted on the damping $\left(k_{I}\right)$ and $\left(k_{2}\right)$.
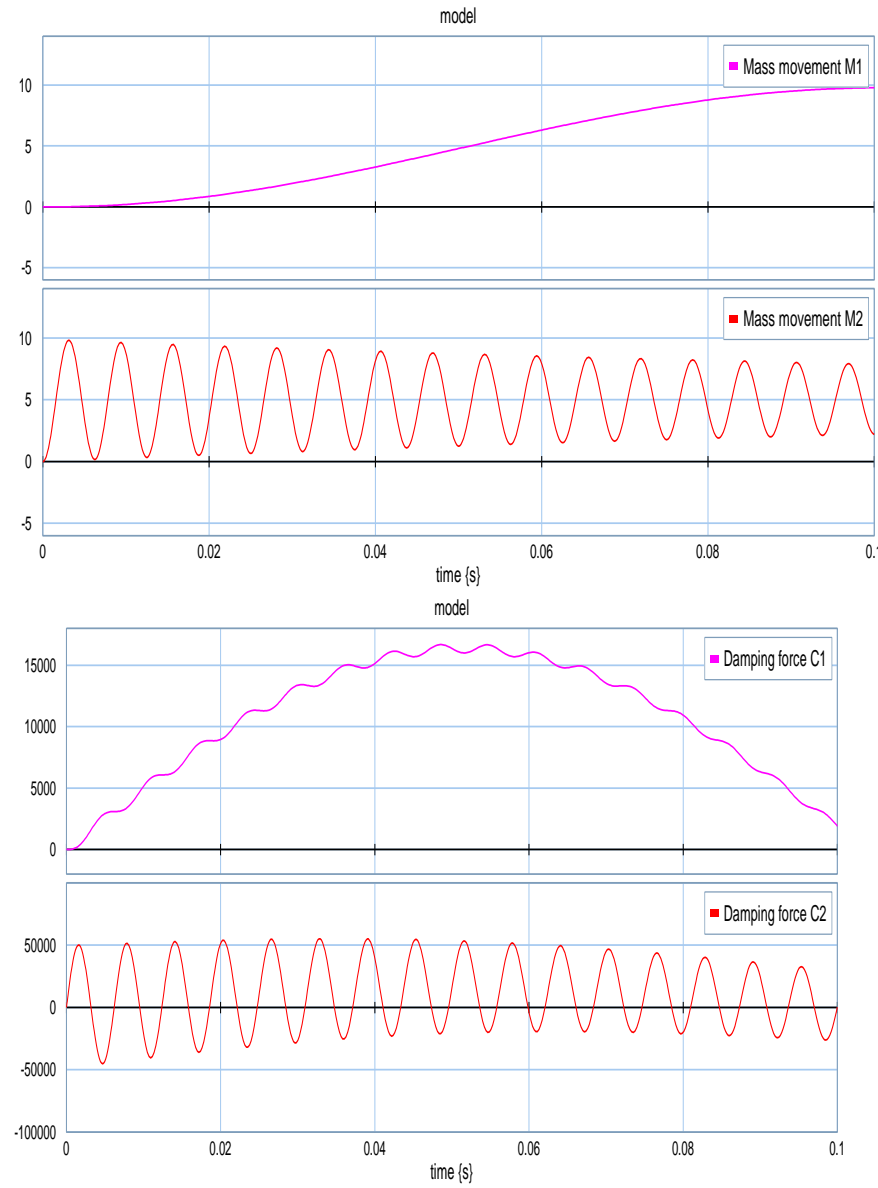

Fig. 6: a) Evolution of the speed on the masse $\left(M_{l}\right.$ and $\left.M_{2}\right)$, b) Evolution the Strengths of the damping $\left(k_{1}\right.$ and $\left.k_{2}\right)$

\subsection{Determination of Residue Equations}

- For example, the junction $1_{1}$ gives us as equation:

$$
r_{1}=e_{1}-e_{2}-e_{3}
$$

According to these relations, one can deduce the residual equation $r_{1}$ :

$r_{1}=-M_{1} \cdot g-M \frac{d D f_{1}}{d t}-D e_{1}$

- The junction $0_{1}$ gives us as equation:

$$
r_{2}=f_{7}-f_{3}-f_{4}
$$

According to these relations, one can deduce the residual equation $r_{2}$ :

$r_{2}=D f_{2}-D f_{1}-\frac{1}{f_{1}} D e_{1}-\frac{k_{1}}{f_{1}} \int D f_{2}-D f_{1}$

- The junction $1_{2}$ gives us as equation:

$r_{3}=e_{10}-e_{9}-e_{8}-e_{7}$

According to these relations, one can deduce the residual equation $r_{3}$ :

$r_{3}=D e_{2}-M_{2} \cdot g-M_{2} \frac{d D f_{2}}{d t}-D e_{1}$ 
- The junction $0_{2}$ gives us as equation:

$r_{4}=f_{11}-f_{10}-f_{12}$

According to these relations, one can deduce the residual equation $r_{4}$ :

$r_{4}=v-D f_{2}-\frac{1}{f_{2}} D e_{2}-\frac{k_{2}}{f_{2}} \int v-D f_{2}$

The rows of the fault signature matrix are associated with the set of residues $r_{1}, r_{2}, r_{3}$ and $r_{4}$ the columns with the variables of the possible faults $F j(j=1,2 \ldots, n) . S_{i j}=1$ (or 0 ), if the residue $i$ sensitive (insensitive) to the fault $j$, we obtain the following signatures table 1 :

Table 1: Fault Signatures Matrix for the Vehicular Active Suspension System

\begin{tabular}{|l|c|c|c|c|}
\hline Element/Residue & $r_{1}$ & $r_{2}$ & $r_{3}$ & $r_{4}$ \\
\hline $\mathrm{Se}_{1}: \mathrm{M}_{1} \cdot \mathrm{g}$ & 1 & 0 & 0 & 0 \\
\hline $\mathrm{Se}_{2}: \mathrm{M}_{2} \cdot \mathrm{g}$ & 0 & 0 & 1 & 0 \\
\hline $\mathrm{C}_{1}: 1 / \mathrm{k}_{1}$ & 0 & 1 & 0 & 0 \\
\hline $\mathrm{C}_{2}: 1 / \mathrm{k}_{2}$ & 0 & 0 & 0 & 1 \\
\hline $\mathrm{R}_{1}: \mathrm{f}_{1}$ & 0 & 1 & 0 & 0 \\
\hline $\mathrm{R}_{2}: \mathrm{f}_{2}$ & 0 & 0 & 0 & 1 \\
\hline $\mathrm{I}_{1}: \mathrm{M}_{1}$ & 1 & 0 & 0 & 0 \\
\hline $\mathrm{I}_{2}: \mathrm{M}_{2}$ & 0 & 0 & 1 & 0 \\
\hline $\mathrm{Df}_{1}$ & 1 & 1 & 0 & 0 \\
\hline $\mathrm{Df}_{2}$ & 0 & 1 & 1 & 1 \\
\hline $\mathrm{De}_{1}$ & 1 & 1 & 0 & 0 \\
\hline $\mathrm{De}_{2}$ & 0 & 0 & 1 & 1 \\
\hline & & & & \\
\hline
\end{tabular}

Figure 7 shows the evolution of residues $r_{1}, r_{2}, r_{3}$ and $r_{4}$ as a normal function. The pitches of the residues converge towards zero under normal operating conditions.

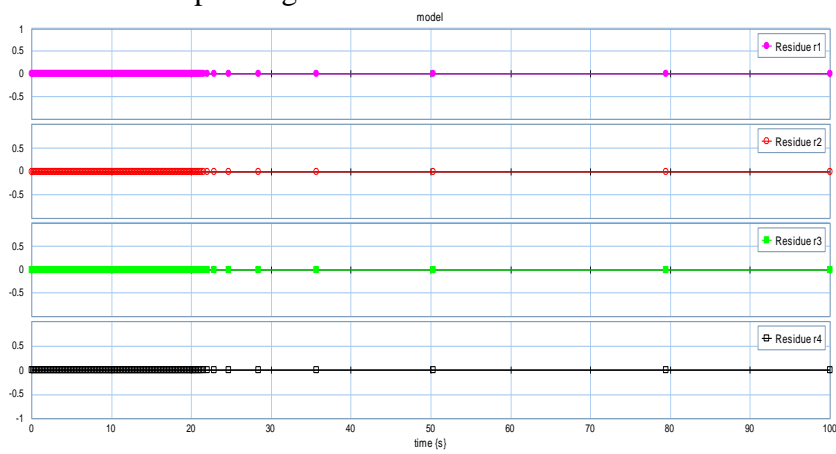

Fig.7: Evolution of residues $r_{1}, r_{2}, r_{3}$ and $r_{4}$ as a normal function

\subsection{Simulation Active Suspension System with Fault}

$\checkmark$ fault on the damper $\left(k_{l}\right)$

Figure 8 show that, when a fault occurs on the damper $k_{l}$, the patterns of the residues $r_{1}, r_{2}, r_{3}$ and $r_{4}$ have non-zero average values, hence these residues are sensitive to this defect and this is confirmed by the theoretical results presented in table 1 .

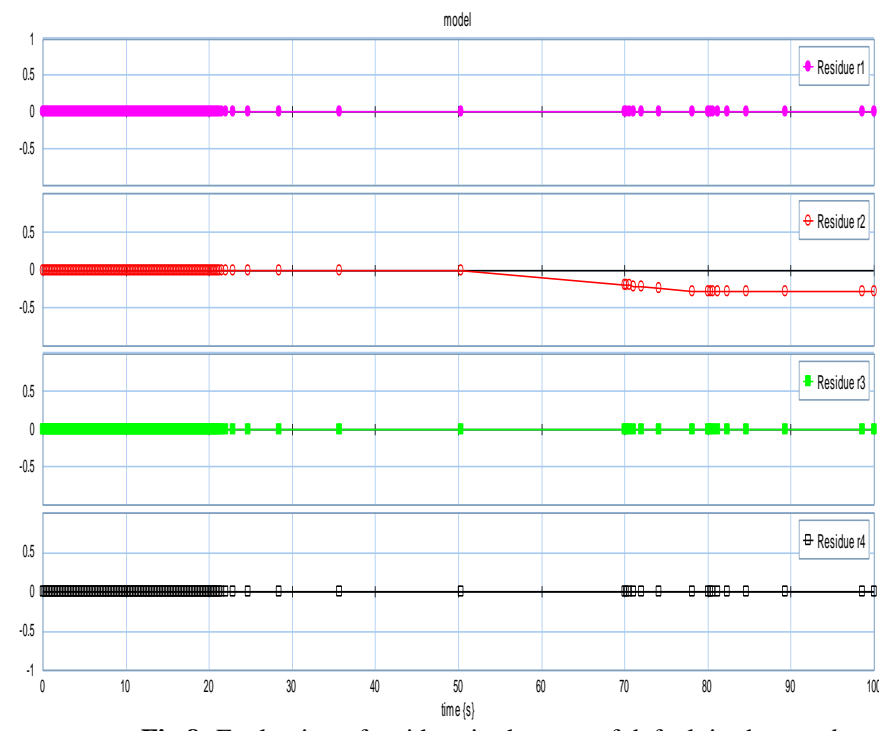

Fig.8: Evaluation of residues in the case of default in damper $k_{l}$

Figure 9 shows that when a defect occurs on the damper $k_{l}$, the variation of the forces exerted on the shock absorbers $k_{1}$ and $k_{2}$ varies at the same time.

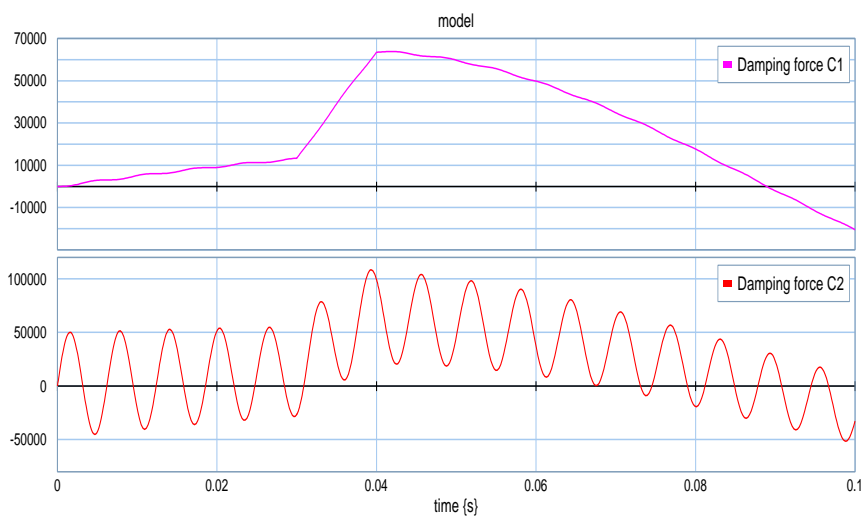

Fig. 9: Evolution of the speed on the masse and the Strengths of the damping $\left(k_{1}\right)$ and $\left(k_{2}\right)$ in the case of default in damper $k_{1}$

fault on the damper $\left(k_{2}\right)$

Figure 10 show that, when a fault occurs on the damper $k_{2}$, the patterns of the residues $r_{1}, r_{2}, r_{3}$ and $r_{4}$ have non-zero average values, hence these residues are sensitive to this defect and this is confirmed by the theoretical results presented in table 1 .

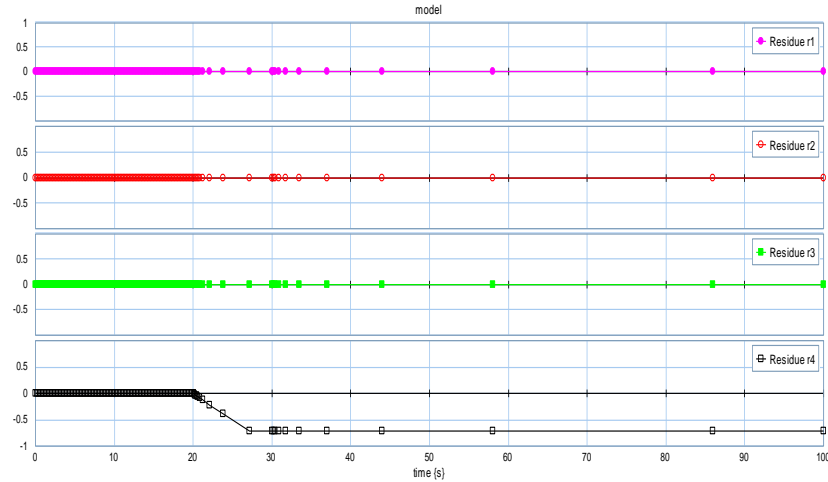

Fig.10: Evaluation of residues in the case of default in damper $k_{2}$

Figure 11 shows that when a defect occurs on the damper $k_{2}$, the change in the forces exerted on the dampers $k_{1}$ and $k_{2}$ varies at the same time. 


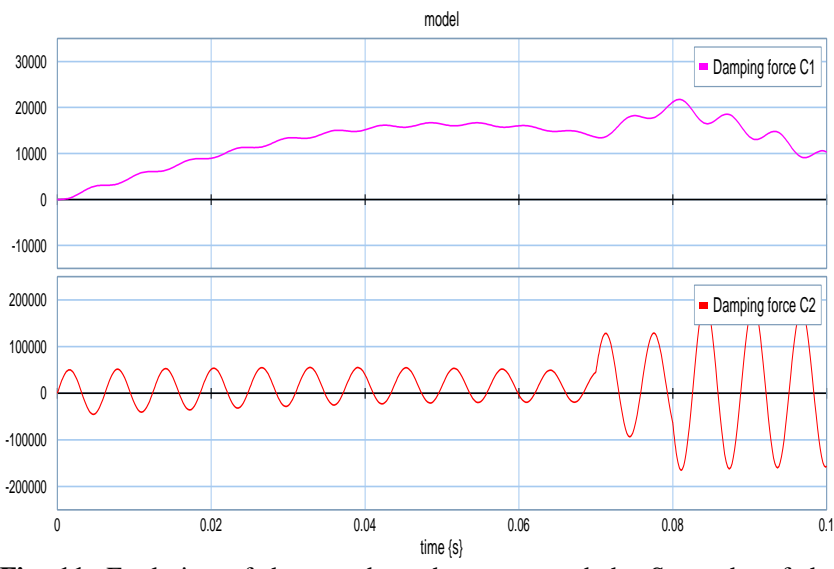

Fig. 11: Evolution of the speed on the masse and the Strengths of the damping $\left(k_{1}\right)$ and $\left(k_{2}\right)$ in the case of default in damper $k_{2}$

\subsection{Robust diagnosis by bond graphs}

Figure 12 shows the BG-LFT model of the hydraulic system.

- For example, the junction $1_{1}$ gives us as equation

$R_{d 1}=e_{1}-e_{2}-e_{3}+Y s_{1}$

According to these relations, one can deduce the residual equation $R_{l}$ :

$R_{d 1}=M_{1} \cdot g \quad M_{1} \frac{d S S f_{1}}{d t} \quad S S e_{1}+Y_{s 1}+w_{M 1}$

The equation consists of two parts: the first part is the normal evolution of the residual $r_{I n}$ and the second part represents the residual uncertainty related to the evolution of the parameters $d_{l}$ :

$$
\begin{aligned}
& R_{d 1}=r_{1 n}+d_{1} \\
& r_{l n}=M_{1} \cdot g \quad M_{1} \frac{d S S f_{1}}{d t} \quad S S e_{1} \\
& d_{1}=Y_{S 1}+w_{M I}
\end{aligned}
$$

- The junction $0_{1}$ gives us as equation:

$R_{d 2}=f_{7}-f_{3}-f_{4}$

According to these relations, one can deduce the residual equation $r_{2}$ :

$R_{d 2}=\operatorname{SSf}_{2} \quad \operatorname{SSf}_{1} \quad \frac{1}{f_{1}} \operatorname{SSe}_{1} \quad \frac{k_{1}}{f_{1}} \quad \oint S f_{2} \quad S S f_{1}+w_{f 1}+w_{\frac{1}{k l}}$

The equation consists of two parts: the first part is the normal evolution of the residual $r_{2 n}$ and the second part represents the residual uncertainty related to the evolution of the parameters $d_{2}$.

$$
\begin{aligned}
& R_{d 2}=r_{2 n}+d_{2} \\
& r_{2 n}=S S f_{2} \quad S S f_{1} \quad \frac{1}{f_{1}} S S e_{1} \quad \frac{k_{1}}{f_{1}} \quad \oiint S f_{2} \quad S S f_{1} \\
& d_{2}=w_{f 1}+w_{\frac{1}{k l}} \\
& \text { - The junction } 1_{2} \text { gives us as equation: }
\end{aligned}
$$

$R_{d 3}=e_{10}-e_{9}-e_{8}-e_{7}$

According to these relations, one can deduce the residual equation $R_{3}$ :

$R_{d 3}=S S e_{2} \quad M_{2} \cdot g \quad M_{2} \frac{d S S f_{2}}{d t} \quad S S e_{1}+w_{M 2}$

The equation consists of two parts: the first part is the normal evolution of the residual $r_{3 n}$ and the second part represents the residual uncertainty related to the evolution of the parameters $d_{3}$.

$$
\begin{aligned}
& R_{d 3}=r_{3 n}+d_{3} \\
& r_{3 n}=S S e_{2} \quad M_{2} \cdot g \quad M_{2} \frac{d S S f_{2}}{d t} \quad S S e_{1}+w_{M 2} \\
& d_{3}=w_{M 2}
\end{aligned}
$$

- The junction $\mathrm{O}_{2}$ gives us as equation:

$R_{d 4}=f_{11}-f_{10}-f_{12}+Y_{s 2}$

According to these relations, one can deduce the residual equation $r_{4}$ :

$$
R_{d 4}=v \quad S_{S} f_{2} \frac{1}{f_{2}} \operatorname{SSe}_{2} \frac{k_{2}}{f_{2}} \oint S S f_{2}+Y_{s 2}+w_{f 2}+w_{\frac{1}{k 2}}
$$

The equation consists of two parts: the first part is the normal evolution of the residual $r_{4 n}$ and the second part represents the residual uncertainty related to the evolution of the parameters $d_{4}$ :

$$
\begin{aligned}
& R_{d 4}=r_{4 n}+d_{4} \\
& r_{4 n}=v \quad S S f_{2} \quad \frac{1}{f_{2}} S S e_{2} \quad \frac{k_{2}}{f_{2}} \quad \oint \quad S S f_{2} \\
& d_{4}=Y_{s 2}+w_{f 2}+w_{\frac{1}{k 2}}
\end{aligned}
$$

\section{Conclusion}

The choice of the LFT form for the modeling of parametric uncertainties with the hop graphs on a nano-technology system made it possible to use a single tool for the systematic generation of indicators of formal uncertainty. These parametric uncertainties are explicitly introduced on the physical model thanks to its graphic architecture, which makes it possible to clearly show on the model their origins.

From a theoretical point of view, the uncertain analytical redundant relations (ARRs) generated are well structured, showing separately the energy contribution of the uncertainties to the default indicators and facilitating their evaluations in the decision step. From a practical point of view, the fields of application of this method are very vast thanks to the energy and multi-physical aspect of the bond graphs, and to the LFT form used to model the influence of the uncertainties on the system. The developed procedure is implemented on a 20SIM software tool [27]. 
influence of the uncertainties on the system. The developed procedure is implemented on a 20SIM software tool [27].

\section{Acknowledgment}

We thank the National School of Engineers of Tunis, University of Tunis El Manar to support our work.

\section{References}

[1] H.M. Paynter. « Analysis and design of engineering systems», M.I.T.Press, 1961.

[2] D.C. Karnopp. and R.C. Rosenberg. «Systems Dynamics: a Unified Approach », Mac Graw Hill, 1983.

[3] R.C. Rosenberg. "Introduction to physical System Dynmics», Series in mechanical engineering, Mac Graw Hill, 1983

[4] M. Tagina. and G. Dauphin, Tanguy. « La méthodologie bond graph Principes et applications», Centre de Publication Universitaire, 2003.

[5] S. Benmoussa, B. Ould-Bouamama, R Merzouki. «Bond Graph Approach for Plant Fault Detection and Isolation: Application to Intelligent Autonomous Vehicle». Automation Science and Engineering, IEEE Transactions on, vol.11, no.2, pp.585-593, April 2014.

[6] A.S. Boudaoud, M. Khemliche, B. Ould Bouamama, S. Bacha and L. F. Lavado Villa. «Bond graph modeling and optimization of photovoltaic pumping system: Simulation and experimental results». Simulation Modelling Practice and Theory Journal, Vol. 36, pp. 84103, August 2013

[7] B. Ould Bouamama, R. El Harabi, M. N. Abdelkrim and M.K Bengayed. "Bond Graphs for diagnosis of Chemical Processes». Computers \& Chemical Engineering, vol. 36, pp. 301-324, 2012.

[8] B. Ould Bouamama, G. Dauphin-Tanguy. «Modelisation Bond Graph Element de base pour l'energetique». Technique de l'ingenieur, BE 8 280, 2005.

[9] B. Ould Bouamama. «Fault detection and isolation of smart actuators using Bond graph and external models». In Control Engineering Practice, Volume 13, $\mathrm{N}^{\circ}$ 2, pp. 159-175, 2004.

[10] B. Ould Bouamama, M. Bayart, K. Medjaher, B. Conard. «FDI of smart actuators using bond graphs and externalmodels». submitted to Control Engineering Practice (CEP) journal, 2002.

[11] B. Ould Bouamama, G. Dauphin-Tanguy, M. Straoswcki, F. Busson. F. «Bond Graph Technique as a Decision-Making Tool in Supervision Systems». HKK Conference \& Symposium in Graph Theoretic \& Entropy Methods in Engineering, University of Waterloo, June 13-15, p 91-97, 1999.

[12] Dauphin-Tanguy, G. 2000. «Les Bond Graphs ». 1st Edn, HERMES Science Publications, Paris, ISBN-10: 2-7462-0158-5.

[13] Henry, D. and A. Zolghari, 2006. Norm-based design of robust FDI schemes for uncertain systems under feedback control: Comparison of two approaches. Control Eng. Pract., 14: 1081-1097. DOI: 10.1016/j.conengprac. 2005.

[14] Han, Z., W. Li and S.L. Shah, 2002. Fault detection and isolation in the presence of process uncertainties. Proceedings of the 15th IFAC World Congress, (WC'02), pp: 1887-1892.

[15] Redheffer. R, 1994. On a certain linear fractional transformation. EMJ. Maths Phys., 39: 269-286.

[16] Alazard. D, C. Cumer, P. Apkarian, M. Gauvrit and G. Fereres, 1999. Robustesse et Commande Optimale. 1st Edn., CépaduesEditions, Toulouse, ISBN-10: 2854285166, pp: 348.

[17] Oustaloup, A., 1994. La robustesse. 1st Edn. Hermès, ISBN-10 2.86601.442.1.

[18] SallamI. A, Zanzouri. N and B. Ould Bouamama «Robus Supervision of Industrials Systems by Bond Graph and External Models». International Journal of Enhanced Research in Science Technology \& Engineering ISSN: 23197463, Vol. 5 Issue 3, March 2016

[19] Sallami. A, Nadia. Z, Mekki. K. «Robust Fault Diagnosis Observer of Dynamical Systems Modelled by Bond Graph Approach». International Journal of Computer Science and Network Security, vol.12 No.1, January 2012.

[20] Djeziri, M.A., B. Ould Bouamama and R. Merzouki, 2009. «Modelling and robust FDI of steam generator using uncertain bond graph model». J. Process Control, 19: 149-162. DOI 10.1016/j.jprocont.2007.12.009

[21] Djeziri. M.A., 2007. «Diagnostic des systèmes incertains par l'approche bond graph». Thèse de Doctorat, École Centrale de Lille.
[22] Vergé. M and Jaume. D. «Modélisation Structurée des Systèmes avec les Bond Graphs». Editions Technip, Paris, 2004.

[23] Baudouin F. et Lavabre M. «Capteurs: principes et utilisations». Casteilla, 2007

[24] Fraden. J. «Handbook of modern sensor».s. Springer-Verlag, 2004. Voir le $\S 13.2$ pour les capteurs capacitifs, en particulier celui d'humidité. Le chapitre 18 est consacré aux matériaux des capteurs, le $\S 18.3$ décrit plus précisé- ment les technologies de fabrication des MEMS.

[25] Viktorovitch. P. «Microsystèmes opto-électromécaniques MOEMS». hermès - Collection Traité EGEM, 2003.

[26] Asch. G. «Acquisition de données; du capteur à l'ordinateur». Dunod, 1999.

[27] Controllab Products, 20-sim version 4.0, http://www.20sim.com 